\title{
Inhaled bronchodilator treatment via the nebuhaler in young asthmatic patients
}

\author{
J B POOL, A GREENOUGH, J G A GLEESON, AND J F PRICE \\ Paediatric Respiratory Laboratory, Department of Child Health, King's College Hospital, London
}

SUMMARY Changes in functional residual capacity and peak flow rate were measured to assess bronchodilator response to terbutaline inhaled via a nebuhaler. In 10 children with asthma, aged 5-7 years, five breaths sufficient to operate the nebuhaler valve resulted in clinically important improvement in both the functional residual capacity and the peak flow rate. In 18 of 22 children, aged 2-5 years, who were too young to have their peak flow rate measured reliably, terbutaline administered via this modified nebuhaler technique was also associated with a clinically important change in functional residual capacity. The results suggest that effective bronchodilation using a nebuhaler can be achieved even in very young children.

Inhaled bronchodilator treatment has advantages compared with oral treatment: the onset of action is more rapid and smaller doses are required so there is less risk of side effects. ${ }^{1}$ It is difficult, however, to give bronchodilator by inhalation to very young asthmatic patients other than by using a nebuliser. A pear shaped spacer device (nebuhaler) has been shown to be an effective means of administering bronchodilators to adults ${ }^{2}$ and older children. ${ }^{3}$ Recently it has been suggested that it is not necessary to follow the manufacturers recommended manoeuvre of two deep inhalations from residual volume, but that five normal breaths of a sufficient magnitude to move the nebuhaler valve resulted in effective administration of treatment. ${ }^{4}$ The latter manoeuvre is easy even for preschool children to perform.

It is difficult to assess bronchodilator response in young children who cannot reliably perform peak flow measurements. The measurement of functional residual capacity, however, is well tolerated and reproducible in children even as young as 2 years of age. ${ }^{5}$ Using this technique we have found that most young asthmatic patients have raised lung volumes ${ }^{6}$ and that nebulised bronchodilator treatment in such young children can be associated with a reduction in the functional residual capacity. ${ }^{7}$

In the present studies we first investigated changes in functional residual capacity and peak flow rate, after bronchodilator treatment via the nebuhaler, in children with asthma who were capable of performing both tests. Secondly, we investigated whether children with asthma who were too young to have their peak flow rate measured reliably had a similar change in functional residual capacity on nebuhaler treatment.

\section{Patients and methods}

Ten consecutive children (nine boys, one girl; mean age $5 \cdot 8$ years, range $5 \cdot 3-6 \cdot 9)$ were enrolled into the first part of the study. Twenty two consecutive preschool children (14 boys, eight girls; mean age 3.6 years, range 2.5-4.9) were enrolled into the second part of the study. All the children were attending the paediatric clinic at King's College Hospital for the treatment of perennial asthma. Asthma was diagnosed after three separate episodes of wheezing and a clinical response to oral bronchodilator treatment. All children were receiving $\beta$ adrenergic stimulants, and in addition 13 were being treated with theophylline orally and one of the older children inhaled steroids.

Permission for this study was granted by the King's College Hospital Ethics Committee. Informed parental consent was obtained.

All the children were studied in the paediatric respiratory laboratory at King's College Hospital; $\beta$ adrenergic stimulants were omitted at least four hours before the measurement were taken.

On arrival the child's functional residual capacity was measured and when possible the peak flow rate. The child then received $500 \mu \mathrm{g}$ terbutaline via a metered dose aerosol through the nebuhaler. The canister was compressed to release a dose of $250 \mu \mathrm{g}$. The child then took five breaths of sufficient 
magnitude to move the nebuhaler valve; this manoeuvre was then repeated. After 10 minutes measurements of functional residual capacity and peak flow rate (where possible) were repeated. As an assessment of symptomatic response, 10 minutes after bronchodilator treatment the child was asked if he or she felt less wheezy, the same, or worse. Children who failed to show either a clinically important change in either measurement (see below) were asked to reattend the laboratory at a later date for retesting.

\section{RESPIRATORY FUNCTION MEASUREMENTS}

Functional residual capacity was measured by helium gas dilution. The measurement was made with the child in a sitting position breathing through a water sealed spirometer (Gould Pulmonet 3 ) via a face mask. Equilibration was assumed when there had been a change of less than $20 \mathrm{ml}$ in functional residual capacity over a 30 second period. ${ }^{6}$ In eight other young children with asthma (age 2-5 years) six measurements of functional residual capacity had been obtained; there was a mean coefficient of variation of $4 \%$. Thus from these data and our previous results ${ }^{7}$ a clinically important change in functional residual capacity was designated as greater than $8 \%$ of predicted for height. Peak flow rate was measured by a Wright's Peak Flow minimeter and reported as the best of three separate measurements before and after bronchodilator treatment. Changes greater than $10 \%$ predicted for height were used as evidence of an important bronchodilator response.

All lung function measurements were performed by JBP and JG. Recordings of functional residual capacity were coded and subsequently analysed blind of clinical details by AG. Results of both measurements were expressed as a percentage of predicted for height using our own normal ranges for functional residual capacity ${ }^{5}$ and those of Murray and Cook for peak flow rate. ${ }^{8}$

\section{STATISTICAL ANALYSIS}

Bronchodilator response in both functional residual capacity and peak flow rate were assessed for significance using a paired Student's $t$ test. The relation between changes in these measurements to the degree of hyperinflation before bronchodilator treatment was assessed for significance using Spearman's correlation.

\section{Results}

All but one child admitted to feeling better after the administration of terbutaline by the modified nebuhaler technique. In all 10 older children there was a significant change in both measurements: an increase in peak flow rate and a decrease in functional residual capacity $(\mathrm{p}<0.01)$ (fig 1$)$. The response, however, was variable, with a mean change in peak flow rate of $38.5 \%$ (range $10-65 \%$ ) and mean change in functional residual capacity of $18.9 \%$ (range $8-44 \%$ ). Sixteen of the 22 younger asthmatic patients showed a reduction in functional residual capacity after bronchodilator treatment, $(p<0.01)$ (fig 2). In all children the change in peak flow rate and functional residual capacity correlated $(p<0.01)$ with the measurement of functional residual capacity before bronchodilator treatment $(\mathrm{r}=-0.78$ peak flow rate, $r=0.49$ functional residual capacity). Two young asthmatic patients showed an increase in functional residual capacity after bronchodilator treatment: $22 \%$ and $24 \%$ change, respectively. Both these children, though having frequent severe asthma attacks, had functional residual capacities

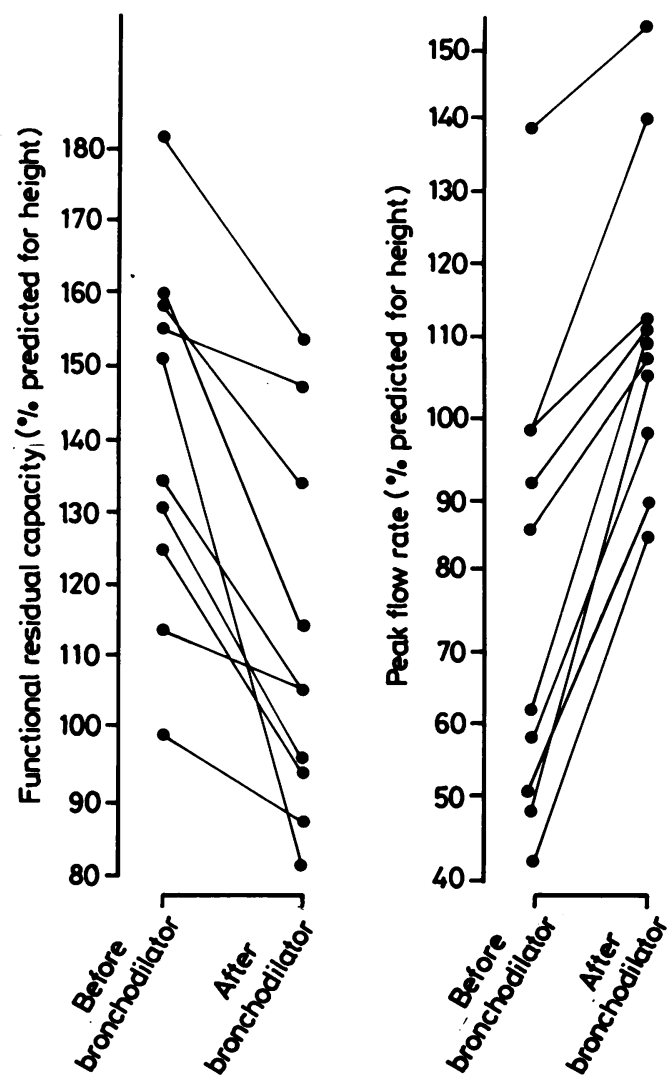

Fig 1 Functional residual capacity and peak flow rate before and after administration of bronchodilator via the nebuhaler in the older children. Each child's results are shown by linked data points. 


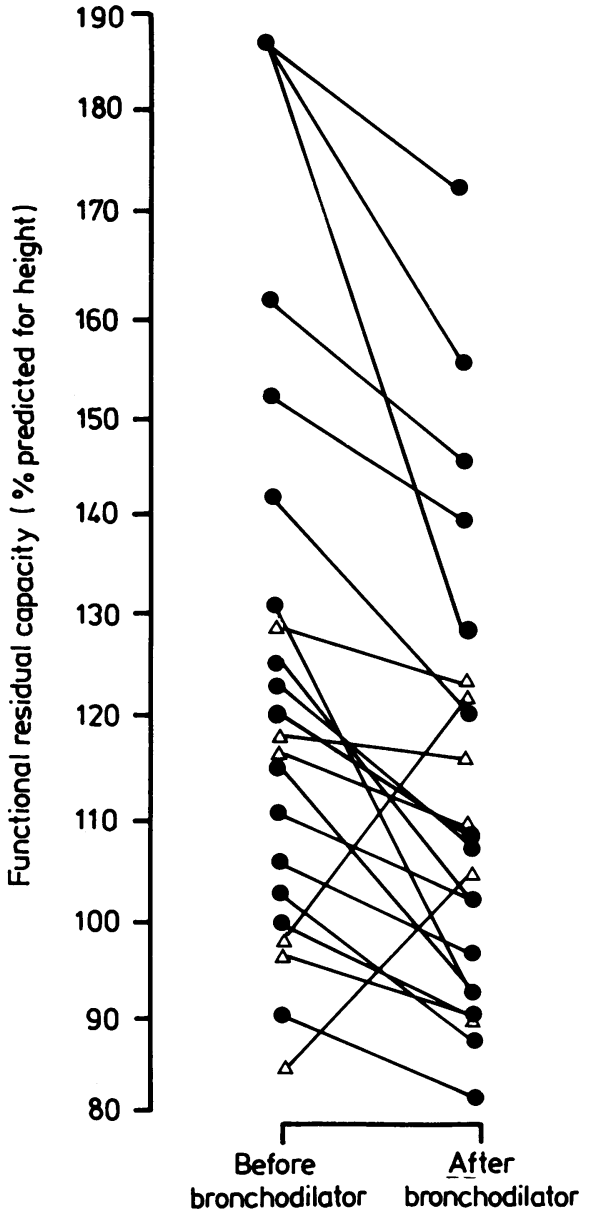

Fig 2 Functional residual capacity before and after administration of bronchodilator in the young children (2-5 years): each child's results are shown by linked data points. Children with a greater than $8 \%$ change in functional residual capacity after bronchodilator treatment $(\mathbf{O})$; children with either a less than $8 \%$ change or an increase in functional residual capacity after bronchodilator treatment $(\triangle)$.

within the normal range before bronchodilator treatment: $101 \%$ and $88 \%$ of predicted for height, respectively.

Of the four young asthmatic patients who failed to show a clinically important change in functional residual capacity $(>8 \%)$ after bronchodilator treatment, three have been recalled for at least two further measurements, and a variable response to treatment has been shown. In one child, there was an increase in magnitude of change in functional residual capacity with repeat testing and in a second a greater than $8 \%$ change in functional residual capacity occurred only when the child was coughing or wheezing, or both, at the time of measurement. The third child, despite being seen on a further three occasions, consistently failed to show a clinically important response to bronchodilator treatment. The fourth child, who showed neither symptom relief or a change in functional residual capacity after bronchodilator treatment, has not been recalled as he no longer attends the asthma clinic as he is symptom free at present.

\section{Discussion}

Bronchodilator treatment is more effectively administered by inhalation. Unfortunately young children have insufficient technical skill to coordinate the operation of a pressurised inhaler and inhalation, and as a consequence nebulisers have become increasingly popular. Nebulisers are large, require an outside power source, and are a relatively inefficient method of administration. Four to eight times the quantity of bronchodilator is necessary when given by a nebuliser to produce an equal effect, ${ }^{9}$ and at most $1 \%$ of the nebulised dose reaches the lung ${ }^{10}$ compared with almost $8 \%$ by a pressurised aerosol. ${ }^{11}$ It has been shown that the attachment of a pear shaped spacer to the pressurised cannister increases the availability of the drug to the patient, even if inhalation is delayed by several seconds; this dispenses with the need to coordinate operation and inhalation. ${ }^{12}$ This mode of delivery is more efficient in that the deposition of the drug in the lungs can be shown to increase to as much as $13 \% .^{11}$

Recently a placebo controlled study has shown that the complicated instructions given by the manufacturers for correct nebuhaler use are not necessary for effective administration of bronchodilator, making this method of treatment more widely applicable. ${ }^{4} \mathrm{~A}$ further study using a tube spacer rather than nebuhaler showed that bronchodilation, assessed by changes in forced expiratory volume in one second, functional residual capacity, and residual volume, was similar if the child inhaled from residual volume or from functional residual capacity; bronchodilation was also similar whether the child inhaled as deeply as possible or only to about half the maximum volume. ${ }^{13}$ The present study confirms that in asthmatic children older than 5 years terbutaline given by modified nebuhaler technique provides effective bronchodilator treatment. The changes in peak flow, confirming a bronchodilator response, were associated with changes in functional residual capacity. In a previous study changes in functional residual capacity were shown in response to salbutamol that was given 
via a nebuliser and these changes were associated with symptom relief. Thus it was felt that the effectiveness of this treatment technique could be assessed reliably by measuring changes in functional residual capacity in children too young to perform peak flow measurements but in whom inhalation by nebuhaler might confer numerous advantages.

In only 18 of the 22 young children tested was there a clinically important change in functional residual capacity. In two of these 18 children there was an increase in functional residual capacity after bronchodilator treatment. Both these children, though having frequent severe asthma attacks, had low functional residual capacities before bronchodilator treatment. A possible explanation for these results was that the children had such severe asthma that there was a limitation of gas mixing. The measurement technique showed that both children had a relatively low functional lung volume before bronchodilator treatment that increased after treatment and this was associated with symptom relief. Of the four children who showed no clinically important change in functional residual capacity, one was probably incorrectly included in this study as he no longer had asthmatic symptoms and thus would have been unlikely to respond to bronchodilator. Lack of a clinically important change in functional residual capacity in the other three children could be explained in two ways: firstly, an insensitivity of the measurement technique or secondly, failure of the treatment technique. It has been suggested that absolute functional residual capacity may not be a true guide to the amount of trapped gas and thus validity of measurements of functional residual capacity in determining hyperinflation could not be anticipated. ${ }^{14} \mathrm{We}$ have, however, previously shown that functional residual capacity is raised in young asthmatic patients and that the degree of this rise is related to the severity of the asthma as judged by the amount of treatment required. ${ }^{6}$ We have also shown that in response to nebulised salbutamol during acute asthma attacks there is a reduction in functional residual capacity and a lessening of hyperinflation, which is associated with an appreciable improvement in compliance. This, plus evidence from the first part of our study that clinically important changes in functional residual capacity were always associated with a similar response in peak flow rate, suggests the measurement technique can reliably detect bronchodilator response. Thus the explanation for the three children in whom a clinically important change in functional residual capacity was not shown after bronchodilator treatment seems likely to be a failure of method of treatment administration. This hypothesis is supported by the increase in magnitude of change in functional residual capacity after bronchodilator treatment that was shown in one child on repeat visits; this suggests a learning process.

From these results we would suggest that bronchodilator given by this modified nebuhaler technique may be an effective form of treatment for young asthmatic children. We would also propose that a change in functional residual capacity may be used in young children, who are too young to perform peak flow rate, to confirm bronchodilator response.

Sister JB Pool and JGA Gleeson are supported by Children Nationwide Medical Research Fund. We thank Mrs Angela McPherson for secretarial help.

\section{References}

' Asthma: clinical patterns and management. In: Phelan PD, Landau LI, Olinsky A, eds. Respiratory illness in children. Oxford: Blackwell Scientific Publications, 1982:184.

2 Stander J, Hidinger K-G. Terbutaline aerosol from a metered dose inhaler with a 750-ml spacer or as a nebulised solution. Respiration 1983;44:237-40.

${ }^{3}$ Freelander M, Van 'Asperen PP. Nebuhaler versus nebuliser in children with acute asthma. $B r$ Med $J$ 1984;288:1873-4.

${ }^{4}$ Gleeson JGA, Price JF. Nebuhaler technique. Br J Dis Chest (in press)

5 Greenough A, Stocks J, Nothen U, Helms P. Total respiratory compliance and functional residual capacity in young children. Pediatr Pulmonol 1986;2:321-7.

6 Greenough A, Pool J, Loftus BG, Price JP. Abnormalities of lung mechanics in young children. Thorax 1987;42:500-5.

7 Greenough A, Loftus BG, Pool J, Price JF. Response to bronchodilators assessed by lung mechanics. Arch Dis Child 1986;61:1020-3.

${ }^{8}$ Murray AB, Cook CD. Measurement of peak flow expiratory flow rates in 220 normal children from $4.5-18.5$ years of age. J Pediatr 1963;62:186-92.

${ }^{9}$ Birk-Madsen E, Bundgaard A, Hidinger KG. A cumulative dose-response study comparing terbutaline pressurised acrosol via a pear shaped spacer and terbutaline in a nebulised solution. Eur J Clin Pharmacol 1982;23:27.

10 Asmundsson T, Johnson RF, Kilburn KH, Goodrich JK. Efficiency of nebulisers for depositing saline in human lung. $\mathrm{Am}$ Rev Respir Dis 1973;108:506-12.

$"$ Newman S, Pavia D, Clarke S. Deposition of pressurised aerosols inhaled through extension devices. Am Rev Respir Dis 1981;124:317-24.

12 Moren F. Drug deposition of pressurised inhalation aerosols. 1. Influence of activator tube design. International Journal of Pharmacy 1978;1:205-8.

${ }_{13}$ Pedersen S. Optimal use of tube-spacer aerosols in asthmatic children. Clin Allergy 1985;15:473-8.

14 Desmond KJ, Coates AL, Martin JG, Beandry PH. Trapped gas and airflow limitation in children with cystic fibrosis and asthma. Pediatr Pulmonol 1986;2:128-34.

Correspondence to Dr A Greenough, Department of Child Health, King's College Hospital, Denmark Hill, London SE5 8RX.

Accepted 12 October 1987 